\title{
CONSUMO ALIMENTAR E ATIVIDADE FÍSICA DE ESTUDANTES UNIVERSITÁRIOS DA ÁREA DE SAÚDE
}

\section{FOOD CONSUMPTION AND PHYSICAL ACTIVITIES OF COLLEGE STUDENTS IN THE HEALTH FIELD}

\author{
Claudia Soar ${ }^{1}$ \\ Patricia de Souza e Silva ${ }^{2}$ \\ Janaína Guarino Lira $^{3}$
}

RESUMO: O estilo de vida de universitários pode contribuir para o aparecimento de doenças crônicas não transmissíveis. Hábitos alimentares não saudáveis e reduzida prática de atividade física durante o período da graduação frequentemente se mantêm após a formação universitária. O objetivo deste estudo foi analisar características da alimentação e da atividade física em universitários de uma instituição privada. Estudo transversal realizado por meio de entrevista sobre estilo de vida. Observou-se maior valor médio do índice de massa corporal e maior prevalência de sobrepeso no gênero masculino ( $p<0,05)$. Identificou-se que $64 \%$ da amostra não realizavam atividade física regular e que $70 \%$ entendiam conhecer a própria necessidade energética, contudo, somente 39\% tinham o conhecimento correto sobre sua necessidade de consumo de energia. Conclui-se que a adequada caracterização do consumo alimentar e da prática de atividade física podem contribuir como ferramentas de auxílio na promoção de saúde desse grupo, pois servirão de base para elaboração de educação em saúde no meio universitário.

Palavras-chave: universitários; consumo alimentar; prática de atividade física; sobrepeso.

ABSTRACT: The lifestyle of college students can contribute to the development of non-transmissible chronic diseases. Unhealthy eating habits and reduced physical activity during the undergraduate years are bad practices often kept after college. This study analyzed the eating habits and physical activity of college students in a private institution. This was a cross-sectional study conducted through an interview on lifestyle. The study found that $64 \%$ of the volunteers did not perform any regular physical activities and $70 \%$ confirmed knowing their energy supply needs; however, only 39\% were correct. This leads to the conclusion that the proper characterization of food consumption and physical activities can be tools to promote health in this group, which can serve as the foundation for educating about healthier practices in a university environment.

Keywords: college students; food consumption; physical activities; overweight.

\footnotetext{
${ }^{1}$ Doutora em Saúde Pública - Universidade de São Paulo - USP e professora da Universidade do Vale do Paraíba - Univap. Email: claudiasoar@univap.br.

${ }^{2}$ Graduada em Nutrição - Univap e Nutricionista de Produção no Hospital Municipal Doutor José Carvalho de Florence, São José dos Campos, SP. E-mail: pathys08@hotmail.com.

${ }^{3}$ Graduada em Nutrição - Univap. E-mail: jana.guarino@gmail.com.
} 


\section{INTRODUÇÃO}

Nos últimos anos, têm-se observado aumento de doenças crônicas não transmissíveis (DCNT), como, por exemplo, a obesidade, 0 diabetes $\mathrm{e}$ as doenças cardiovasculares (DCV). Essas doenças figuram como principal causa de mortalidade e incapacidade no mundo, responsável por $59 \%$ dos 56,5 milhões de óbitos anuais (OPAS, 2003).

Recentemente, o Ministério da Saúde publicou resultados preocupantes sobre os índices de sobrepeso e obesidade no Brasil. Por meio do estudo "Vigilância de fatores de risco e proteção para doenças crônicas por inquérito telefônico - VIGITEL 2010", identificou-se aumento nas prevalências de excesso de peso e obesidade, tanto em homens como em mulheres. A prevalência de excesso de peso, em 2006 , era de $47,2 \%$ em homens e $38,5 \%$ em mulheres; já, em 2010, esses valores passaram para 52,1\% e $44,3 \%$. Especificamente, em relação à obesidade, a prevalência em homens era de $11,4 \%$ e passou para $14,4 \%$, em 2010 . No mesmo período, observou-se, em mulheres, aumento de $11,4 \%$ para $15,5 \%$ (BRASIL, 2011).

Há várias evidências de que a alimentação equilibrada e a prática de atividade física regular atuam diretamente na prevenção dessas e de outras doenças (OPAS, 2003). Embora esse conhecimento seja bem difundido, a dieta denominada ocidental, rica em gorduras, açúcares simples, alimentos refinados e reduzida em fibras, bem como o inatividade física, fazem parte do estilo de vida de grande parte da população.

A associação da dieta de alta densidade energética e o sedentarismo podem levar à obesidade, que é um fator determinante para 0 aparecimento de diabetes tipo 2, dislipidemias e hipertensão, além de aumentar o risco de certos tipos de câncer (WHO, 2003). Também, são descritas evidências de que, quanto mais precoce o estabelecimento da obesidade, maiores são os riscos de obesidade na idade adulta (LOWRY et al., 2000).

Entre universitários, têm-se identificado estilo de vida associado ao aparecimento dessas doenças, pois é comum a omissão de refeições, com preferência pelo consumo de lanches rápidos e refeições nutricionalmente desequilibradas, com elevada ingestão de alimentos doces e gordurosos e baixa ingestão de frutas e hortaliças (PETRIBU; CABRAL; ARRUDA, 2009; ANDING; SUMINSKI; BOSS, 2001; DEBATE; TOPPING; SARGENT, 2001; VIEIRA et al., 2001). A vida universitária pode contribuir de diversas formas para práticas de vida não saudáveis. Uma delas diz respeito a deixar a casa dos pais e passar a viver em moradias estudantis, muitas vezes, devido à localização da instituição, o que pode trazer importantes alterações na rotina diária. Por conta das intensas tarefas acadêmicas, pode haver falta de tempo para realizar refeições completas, bem como atividade física regular, além do estabelecimento de novos comportamentos e relações sociais (MATTOS; MARTINS, 2000; MARCONDELLI; COSTA; SCHMITZ, 2008). Para Vieira et al. (2001), os maus hábitos alimentares podem sugerir indícios de compulsão alimentar em alguns alunos que, ansiosos, podem transformar a alimentação em "válvula de escape" para as situações de estresse físico e mental.

Muitos hábitos alimentares adquiridos por estudantes durante os anos cursados 
nas universidades continuam na idade adulta, ocorrendo a perpetuação de estilo de vida não saudável (HABERMAN; LUFFEY, 1998).

Dado o exposto, o objetivo deste trabalho foi analisar características da alimentação e da atividade física em universitários de uma instituição privada, como forma de identificar a necessidade de implementação de programas de educação em saúde nessa população.

\section{MATERIAL E MÉTODO}

O trabalho teve um delineamento do tipo transversal, com amostra por livre demanda, desenvolvido no mês de abril de 2010, como parte do programa de monitoria acadêmica. Fizeram parte, da amostra, estudantes universitários da área da saúde de uma universidade particular da cidade de São José dos Campos, SP. Foi realizada entrevista, por meio de questionário, com perguntas sobre idade, sexo, peso atual, altura, prática de atividade física e conhecimento sobre necessidade diária de energia, todas as respostas auto-relatadas.

Para classificação do estado nutricional, utilizou-se o índice de massa corporal (IMC), tendo como critério de classificação os valores descritos pela Organização Mundial da Saúde (WORLD HEALTH ORGANIZATION, 1995).

Foi considerada prática de atividade física regular quando o entrevistado relatou realizar atividades pelo menos 3 (três) vezes na semana, de, no mínimo, 30 (trinta) minutos.

No caso de variável conhecimento sobre necessidade diária de energia, foi calculada a necessidade individual, conforme equação da FAO (FAO/WHO/UNU, 1985) e considerado correto conhecimento quando o indivíduo informou um valor que variava em até \pm 400 calorias daquele estimado pela equação.

Aplicou-se teste $\mathrm{T}$ de Student, para verificação de diferença de médias e teste Qui-quadrado para identificação de diferença na prevalência, especificamente, de sobrepeso. O nível de significância adotado foi de $5 \%$.

Os demais resultados foram apresentados de forma descritiva.

\section{RESULTADOS}

Foram entrevistados 61 estudantes da área da saúde dos cursos de Enfermagem, Biomedicina, Farmácia, Fisioterapia, Nutrição, Odontologia e Terapia Ocupacional, sendo 54 universitárias e 7 universitários.

A idade média foi $21( \pm 7,21)$ anos, sendo de $21( \pm 7,59)$ anos para o gênero femino e $24( \pm 4,98)$ anos para o gênero masculino. O IMC médio foi de $22,8( \pm 3,76)$ $\mathrm{kg} / \mathrm{m}^{2}$, sendo de $22( \pm 3,65) \mathrm{kg} / \mathrm{m}^{2}$, no gênero feminino, e 26,3 $( \pm 2,62) \mathrm{kg} / \mathrm{m}^{2}$ no gênero masculino, estatisticamente maior do que o verificado em universitárias (Tabela 1). 
Tabela 1 - Valores médios e desvio padrão das variáveis idade e índice de massa corporal (IMC) de estudantes da área da saúde de uma universidade privada de São José dos Campos, SP, 2010

\begin{tabular}{ccccc}
\hline \multicolumn{1}{c}{ Variável } & $\begin{array}{c}\text { Todos } \\
\mathbf{n = 6 1}\end{array}$ & $\begin{array}{c}\text { Feminino } \\
\mathbf{n}=\mathbf{5 4}\end{array}$ & $\begin{array}{c}\text { Masculino } \\
\mathbf{n = 7}\end{array}$ & Valor de $\mathbf{p}$ \\
\hline Idade (em anos) & 21 & 21 & 24 & 0,771 \\
& $( \pm 7,21)$ & $( \pm 7,59)$ & $( \pm 4,98)$ & \\
IMC $\left(\mathrm{kg} / \mathrm{m}^{2}\right)$ & 22,8 & 22 & 26,3 & 0,001 \\
& $( \pm 3,76)$ & $( \pm 3,65)$ & $( \pm 2,62)$ & \\
\hline
\end{tabular}

Em relação ao estado nutricional, foi estaticamente maior no gênero masculino identificado baixo peso somente no gênero (Tabela 2).

feminino. Já, a prevalência de sobrepeso foi

Tabela 2 - Classificação do estado nutricional de estudantes de uma universidade privada de São José dos Campos-SP, conforme índice de massa corporal. 2010

\begin{tabular}{lcccccc}
\hline Classificação do estado nutricional & \multicolumn{2}{c}{ Todos } & \multicolumn{2}{c}{ Feminino } & \multicolumn{2}{c}{ Masculino } \\
\hline & $\mathrm{n}$ & $\%$ & $\mathrm{n}$ & $\%$ & $\mathrm{n}$ & $\%$ \\
Baixo peso & 4 & 6,5 & 4 & 7,4 & 0 & 0 \\
Eutrofia & 44 & 72,2 & 41 & 76 & 3 & 43 \\
Sobrepeso & 13 & 21,3 & 9 & 16,6 & 4 & 57 \\
Valor de $\mathrm{p}$ & & & & $\mathrm{p}=0,015$ & \\
\hline
\end{tabular}

Conforme Fig. 1, a prática de atividade universitários entrevistados sejam física regular foi observada em $36 \%$ da sedentários. amostra, sugerindo que $64 \%$ dos

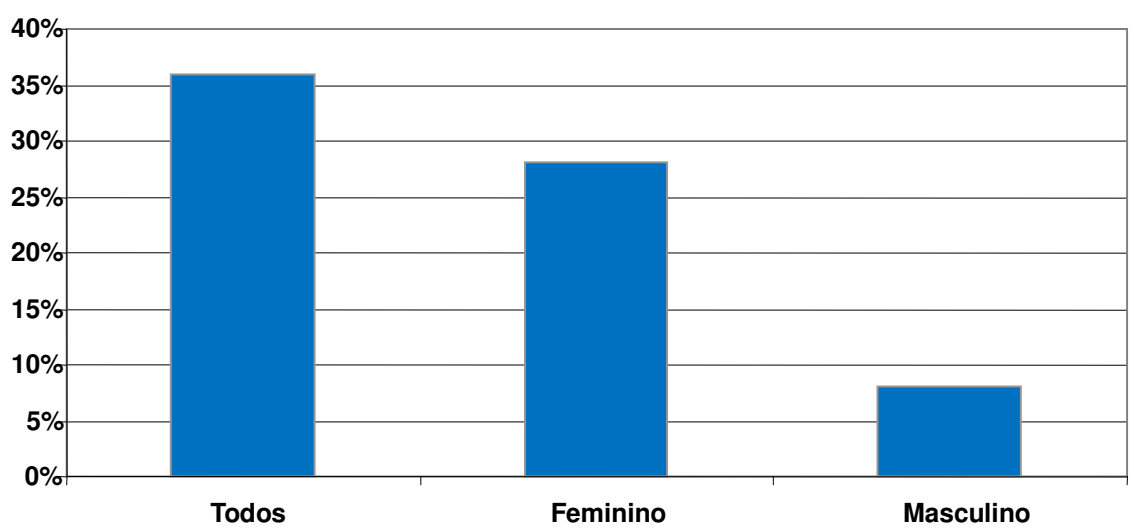

Fig. 1 - Prática de atividade física entre estudantes de uma universidade privada de São José dos Campos - SP, 2010.

A maioria dos entrevistados, $70 \%$, tinham o conhecimento correto, ou seja, relatou conhecer sua necessidade diária de informaram um valor que não refletia a real calorias (Fig. 2), porém, menos da metade, necessidade (Fig. 3). 


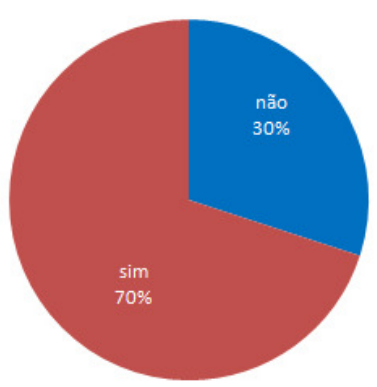

Fig. 2 - Auto-relato de conhecimento sobre a necessidade energética diária entre estudantes de uma universidade privada de São José dos Campos - SP, 2010.

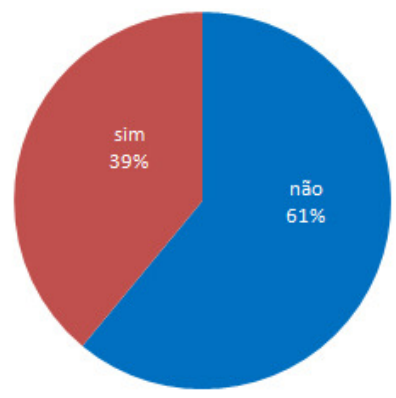

Fig. 3 - Conhecimento correto sobre a necessidade energética diária entre estudantes de uma universidade privada de São José dos Campos - SP, 2010.

\section{CONCLUSÃO E DISCUSSÃO}

A presença de baixo peso no gênero feminino deve ser melhor investigada. Penz et al. (2008) encontraram dados semelhantes e sugeriram que pode ocorrer preocupação com a imagem corporal entre alunos de cursos de saúde, especialmente de Nutrição, composto na sua grande maioria por mulheres.

A maior prevalência de sobrepeso no gênero masculino é questionável, pois foi observado que todos os homens incluídos no estudo eram praticantes de atividade física regular e, possivelmente, com quantidade de massa corporal magra adequada, mesmo com IMC acima do recomendado. Essa consideração é importante no sentido de apontar uma das limitações do estudo, uma vez que não estavam previstas, no estudo, outras formas de avaliação do estado nutricional, como, por exemplo, determinação de composição corporal pela avaliação de dobras cutâneas.

Além disso, os resultados que descrevem o estado nutricional devem ser interpretados com cautela, pois, embora a amostra tenha sido composta, na sua maioria, por indivíduos adultos, fizeram parte da amostra alguns estudantes com idade menor de 18 anos, tendo como recomendação uma classificação de estado nutricional diferente do adulto. No caso de adolescentes, é recomendado considerar-se a maturidade sexual na avaliação do estado nutricional, contudo o local da coleta de dados, salão de entrada de um prédio da Faculdade de Ciência da Saúde, não permitia essa análise. Há, também, uma limitação em relação ao tamanho amostral total e diferença entre os gêneros.

Quando comparado a outros estudos com universitários, foi encontrado índice preocupante de sedentarismo, pois identificou-se, na literatura, uma variação entre $42 \%$ e $60 \%$ (MARCONDELLI; COSTA; SCHMITZ, 2008; PETRIBU; CABRAL; ARRUDA, 2009); indicando que, em nossa amostra, há um percentual elevado de pessoas em inatividade física, condição favorável ao surgimento de doenças crônicas.

Além dessa condição, observou-se, também, um número reduzido de pessoas que possuem conhecimento correto sobre sua necessidade diária de energia, embora tenham descrito que tinham esse 
conhecimento.

Em função dos resultados encontrados, torna-se necessária intervenção nutricional para promover a alimentação saudável para os estudantes, no sentido de prevenir o aparecimento de DCNT futuras. Muitos dos hábitos alimentares adquiridos por estudantes, durante os anos cursados na universidade, continuam durante a fase adulta. $O$ incentivo para prática regular de atividade física também deve fazer parte das metas de melhoria do estilo de vida dos entrevistados.

Portanto, a importância da correta caracterização da dieta e prática de atividade física, como ferramenta de auxílio na promoção de saúde desse grupo, é de grande relevância, pois servirá de base para a adequada educação nutricional e recomendações de atividade física.

Sugere-se a implementação de um programa de educação em saúde voltado à comunidade acadêmica da instituição estudada.

\section{REFERÊNCIAS}

ANDING, J. D.; SUMINSKI, R. R.; BOSS, L. Dietary intake, body mass index, exercise and alcohol: are college women following the dietary guidelines for Americans? J. am. col. health., v. 49, n. 4, p. 167-71. 2001.

BRASIL. MINISTÉRIO DA SAÚDE. Secretaria de Vigilância em Saúde. Secretaria de Gestão Estratégica e Participativa. Vigitel Brasil 2010: vigilância de fatores de risco e proteção para doenças crônicas por inquérito telefônico. Brasília: Ministério da Saúde, 2011.

DEBATE, R. D.; TOPPING, M.; SARGENT, R. G. Racial and gender differences in weight status and dietary practices among college students. Adolescence, v. 36, n. 144, p. 819-833, 2001.

FAO. WORLD HEALTH ORGANIZATION. ONU. Energy and protein requirements. World Health Organ Tech Rep Ser., Geneva, n. 724, p.1-206, 1985.

HABERMAN, S.; LUFFEY, D. Weighing in college students' diet and exercise behaviors. J. Am. Coll. Health., v. 46, n. 4, p. 189-191.1998.

LOWRY, R. et al. Physical activity, food choice, and weight management goals and practices among US college students. Am. $j$. prev. med., v. 18, n. 1, p. 18-27. 2000.

MARCONDELLI, P.; COSTA, T. H. M.; SCHMITZ, B. A. S.. Nível de atividade física e hábitos alimentares de universitários do $3^{\circ}$ ao $5^{0}$ semestres da área da saúde. Rev. Nutr., Campinas, v. 21, n. 1, Feb. 2008. Available from: $<$ http://www.scielo.br/scielo.php?script=sci_a rttext\&pid=S1415 $52732008000100005 \&$ lng=en\&nrm=iso $>$.

Access on: 22 May 2012. http://dx.doi.org/10.1590/S141552732008000100005.

MATTOS, L. L.; MARTINS, I. S. Consumo de fibras alimentares em população adulta. Rev. saúde pública, São Paulo, v. 34, n. 1, p. 5055, 2000.

ORGANIZAÇÃO PAN-AMERICANA DE SAÚDE. Doenças crônico-degenerativas e obesidade: estratégia mundial sobre alimentação saudável, atividade física e saúde. Brasília, DF: OPAS, 2003.

PENZ, L. R. et al. Risco para desenvolvimento de transtornos alimentares em estudantes de Nutrição. Sci. med., Porto Alegre, v. 18, n. 3, p. 124-128. 2008. 
PETRIBU, M. M. V.; CABRAL, P. C.; nutricional e de saúde de adolescentes ARRUDA, I. K. G. Estado nutricional, recém-ingressos em uma universidade consumo alimentar e risco cardiovascular: pública brasileira. Rev. nutr., Campinas, v. um estudo em universitários. Rev. nutr., 15, n. 3, p. 273-82. 2002.

Campinas, v. 22, n. 6, Dec. 2009. Available from:

WORLD HEALTH ORGANIZATION. Physical status: the use and interpretation of $<$ http://www.scielo.br/scielo.php?script=sci_a rttext\&pid=S1415anthropometry. Geneve: WHO, 1995. (Technical Report Series, n. 854). $52732009000600005 \& \operatorname{lng}=e n \& n r m=i s 0>$.

Access on: 22 May 2012. http://dx.doi.org/10.1590/S141552732009000600005 .

of chronic diseases. Report of a Joint WHO/FAO Expert Consultation. Geneva:

VIEIRA, V. C. R. et al. Perfil socioeconômico, WHO, 2003. (Technical Report Series, n. 916). 